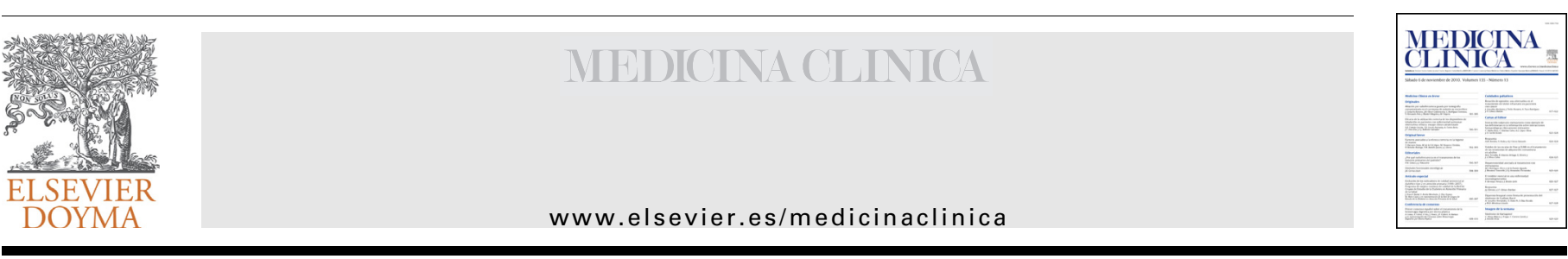

Revisión

\title{
Pronóstico y tratamiento de la boca seca. Revisión sistemática
}

\author{
José López-López*, Enric Jané Salas y Eduardo Chimenos Küstner
}

Departamento de Odontoestomatología, Facultad de Odontología, Universitat de Barcelona, Barcelona, España

I N F O R M A C I Ó N D E L A R T ÍC U L O

Historia del artículo:

Recibido el 16 de enero de 2013

Aceptado el 21 de febrero de 2013

On-line el $\mathrm{xxx}$

\section{Palabras clave}

Boca seca

Hiposalivación

Pronóstico

Tratamiento

Xerostomía

\begin{abstract}
R E S U M E N
No existen protocolos claramente establecidos para el tratamiento de la boca seca. El objetivo de este trabajo es realizar una revisión sistemática de la literatura médica de los últimos 10 años. Hemos utilizado las palabras: "dry mouth», "prognosis», "treatment» y "dentistry». En una primera búsqueda hemos encontrado 1.450 entradas. Con la restricción «clinical trials OR randomized controlled trial OR systemic reviews" se han reducido a 522, de las que 145 eran metaanálisis y revisiones sistemáticas. Se han eliminado las que no eran pertinentes al tema y han quedado reducidas a 53. Posteriormente se descartaron 24 ( 8 no pertinentes, 7 revisiones y 9 opiniones personales). De los 29 artículos analizados, 15 son ensayos clínicos controlados, 2 ensayos no controlados, 4 estudios observacionales, 2 revisiones sistemáticas y 5 revisiones. Los enfermos mejor estudiados son los pacientes con síndrome de Sjögren y los pacientes irradiados. El tratamiento se centra en el etiológico, preventivo, sintomático, de estimulación salival local y sistémicos. Podemos concluir que el tratamiento debe ser individualizado, pudiéndose aplicar sustitutos salivales y diferentes técnicas de estimulación mecánica.
\end{abstract}

(c) 2013 Elsevier España, S.L. Todos los derechos reservados.

\section{Prognosis and treatment of dry mouth. Systematic review}

\begin{abstract}
A B S T R A C T
There are no clearly established protocols for the treatment of dry mouth. The aim of this paper is a systematic review of the literature of the past 10 years using the words "dry mouth", "prognosis", "treatment" and "dentistry». The initial search found 1,450 entries and within the restriction "clinical trials OR randomized controlled trial OR systemic reviews» it has been reduced to 522, which 145 were meta-analysis and systematic reviews. Papers not relevant to the issue were removed reducing the entries to 53. Twenty-four were dismissed ( 8 irrelevant, 7 reviews without adequate information and 9 personal opinions). Of the 29 items tested, 15 were controlled trials, 2 uncontrolled trials, 4 observational studies, 2 systematic reviews and 5 non systematic reviews. The most studied patients were Sjögren's syndrome and the irradiated patients. Treatments are focused on the etiology, prevention, symptomatic, local salivary stimulation and systemic treatments. It can be concluded that treatment must be individualized, salivary substitutes and mechanical stimulation techniques can be applied.
\end{abstract}

(c) 2013 Elsevier España, S.L. All rights reserved.

\section{Introducción}

La calidad y el volumen adecuados de la saliva son necesarios para proteger los tejidos bucales. Muchos pacientes se quejan de boca seca, pero al explorar su cavidad oral, en muchas ocasiones no encontramos signos objetivos que la justifiquen ${ }^{1}$. Clínicamente denominamos xerostomía a la sensación subjetiva de boca seca. Si hay una disminución de las tasas de flujo salival por debajo de 0,1 -

\footnotetext{
* Autor para correspondencia.

Correo electrónico: 18575jll@gmail.com (J. López-López).
}

$0,2 \mathrm{ml} / \mathrm{min}$ en la saliva total en reposo y por debajo de $0,4-0,7 \mathrm{ml} /$ min en la saliva total estimulada, hablamos de hiposialia 0 hiposecreción salival ${ }^{2,3}$.

Aproximadamente el $70 \%$ de la secreción salival procede de las glándulas submandibulares y sublinguales, un $15-20 \%$ procede de las parótidas y un 5-8\% procede de las glándulas salivales menores. La saliva estimulada procede de las parótidas, de secreción serosa, en un $45-50 \%$. La saliva de reposo, secretada por las glándulas de secreción mucosa, es la principal responsable de la lubricación y protección de la mucosa.

Si excluimos la radioterapia y el síndrome de Sjögren (SS), las alteraciones glandulares que se producen con el paso de los años, y

0025-7753/\$ - see front matter (c) 2013 Elsevier España, S.L. Todos los derechos reservados. http://dx.doi.org/10.1016/j.medcli.2013.02.036 
en especial los efectos anticolinérgicos de la medicación en pacientes de edad avanzada, son los principales responsables de una disminución del flujo salival, especialmente de reposo (tabla 1$)^{4}$. Por otro lado, sabemos que si bien en muchas ocasiones la sequedad se relaciona con los hábitos de vida y especialmente con los hábitos alimentarios, existen múltiples factores locales y sistémicos que intervienen en su etiopatogenia (tabla 2).

La clínica de la boca seca dependerá de su intensidad, pero también de la causa de la hiposalivación. Es de esperar que cuando haya una base orgánica irreversible, como puede ser la destrucción del parénquima por radioterapia o enfermedad inmunitaria, el pronóstico será peor y el tratamiento deberá enfocarse de una manera diferente a las situaciones en las que quede reserva glandular o el problema sea temporal. También influirá la vivencia que los pacientes tengan frente a su problema ${ }^{5,6}$.

En el tratamiento es prioritario determinar los factores de riesgo y la etiología mediante una historia clínica detallada. Una vez detectada la causa, esta deberá eliminarse, si es posible, o bien habrá que buscar una estrategia terapéutica paliativa para las consecuencias $^{2,7}$. En la actualidad el tratamiento de la boca seca no tiene un protocolo establecido y las diferentes estrategias se recogen en la tabla 3.

En base a todo lo anteriormente expuesto, en el presente trabajo revisamos la evidencia científica publicada acerca del tratamiento y el pronóstico de la boca seca.

\section{Material y método}

Se realizó una búsqueda desde 1988 a noviembre de 2012 con las palabras clave (MeSH) «dry mouth", "prognosis», "treatment» y "dentistry», incluyendo revisiones sistemáticas, estudios clínicos, estudios descriptivos, series de casos y controles, casos clínicos y revisiones bibliográficas. Dos examinadores independientes revisaron los resúmenes y se descartaron los trabajos irrelevantes o los que hacían referencia a un caso clínico y no aportaban nada nuevo. Luego se estratificaron los artículos de acuerdo con su nivel de evidencia científica usando los strength of recommendation taxonomy ${ }^{16}$. Después, en los ensayos clínicos aleatorizados se evaluó su nivel de calidad usando los criterios JADAD ${ }^{17}$. Posteriormente, en base a la evidencia obtenida se expresaba una opinión del trabajo, ya sea a favor o en contra de aceptar sus conclusiones ${ }^{16}$. Finalmente los autores compararon los resultados y expusieron sus opiniones; si había desacuerdo y este persistía, opinaba un tercer investigador.

\section{Resultados}

La respuesta a la búsqueda «dry mouth AND treatment AND prognosis» mostró 1.450 artículos; 982 si la búsqueda se restringía a 10 años y humanos. Al escoger «clinicial trials OR randomized

Tabla 1

Principales alteraciones detectables en un paciente con xerostomía

Sensación de sequedad bucal
Saliva viscosa, pegajosa
Disgeusia
Olfato alterado
Dificultad para hablar
Dificultad masticatoria
Aumento de caries dentales destructivas
Erosiones dentarias (en forma de cuñas cervicales)
Halitosis
Dolor retroesternal por reflujo gastroesofágico
Boca ardiente (glosodinia, estomatopirosis)
Labios fisurados
Candidiasis persistentes o de repetición
Úlceras orales
Mala adaptación a las prótesis dentales

Sensación de sequedad bucal

Saliva viscosa, pegajosa

Halitosis

Dolor retroesternal por reflujo gastroesofágico

Labios fisurados

Candidiasis persistentes o de repetición

Mala adaptación a las prótesis dentales
Tabla 2

Causas de sequedad bucal

Afectación del parénquima glandular

Enfermedades infecciosas recidivantes o crónicas de las glándulas mayores (bacterias, virus)

Enfermedad autoinmunitaria primaria o secundaria (síndrome de Sjögren), sialoadenosis

Destrucción de tejido glandular tras radioterapia

Disfunción glandular

Debida a deshidratación

Ausencia de estimulación mecánica

Acción de fármacos anticolinérgicos o agonistas

Interacción de fármacos entre sí o con inductores enzimáticos (alcohol, tabaco)

controlled trial OR systematic reviews» se obtienen 436. Dado el elevado número se realizó una nueva restricción con la palabra "dentistry» y se obtuvieron 522 artículos (145 eran metaanálisis y revisiones sistemáticas). Después de leer el resumen, se descartaron 102 por centrarse en aspectos etiológicos, en el tema de la caries, aspectos diagnósticos, tratamientos con implantes en pacientes con boca seca, candidiasis o en el síndrome de boca ardiente. Finalmente se recuperaron a texto completo 49 artículos.

Tabla 3

Sistemática de actuación en el paciente con boca seca

1 Valoración crítica de los fármacos que toma ${ }^{8,9}$

2 Medidas complementarias

Beber 2-31 de agua al día (infusiones, zumos sin azúcares añadidos, leche, etc. $)^{10,11}$

Evitar ambientes desecantes, como el aire acondicionado y calefacción excesivos $^{1}$

Evitar tabaco y alcohol (tópico y sistémico), son secantes e inductores enzimáticos, así como elevadas dosis de cafeína (café, bebidas de cola, etc. $)^{4}$

Evitar y combatir el estrés (mediante ejercicio físico regular) ${ }^{4}$ Higiene bucodental periódica ${ }^{1}$

Visitas frecuentes al dentista para prevenir y tratar lesiones incipientes ${ }^{1,4}$

3 Medidas específicas ${ }^{2}$

Estimular la función remanente de las glándulas salivales, si es posible

Si no hay función salival remanente, contrarrestar las consecuencias negativas con protección y lubricación externa

Tratar precozmente los trastornos asociados a la boca seca (caries, candidiasis, gingivitis, periodontitis, etc.)

Paliar los signos y síntomas de boca seca (sequedad oral, candidiasis, úlceras, dolor, etc.)

Valorar el control de la ansiedad y el estrés si se presentan (ketazolam $15 / 30 \mathrm{mg}$ después de cenar $)^{2}$

4 Estimular la función salival ${ }^{12,13}$

Masticar chicle sin azúcares cariogénicos, de sabores intensos

Chupar pastillas o comprimidos de parecida composición

Masticar alimentos consistentes

Estimulación eléctrica (aparato Biosonics ${ }^{\mathbb{R}}$ )

Acupuntura

5 Administrar sialogogos (estimulantes farmacológicos, con acción agonista colinérgica)

Deben administrarse con precaución, teniendo en cuenta sus contraindicaciones ${ }^{2}$ : anetol tritiona, betanecol, piridostigmina, pilocarpina, cevimelina

6 Sustitutos salivales (deben reunir algunas características: $p H$ fisiológico de sabor agradable, sin ácidos ni azúcares, con antisépticos; de viscosidad similar a la saliva, con iones [ $\mathrm{F}, \mathrm{Ca}, \mathrm{P}, \mathrm{K}, \mathrm{Mg}, \mathrm{Cl}]$ y enzimas [lisozima, peroxidasa]) ${ }^{13}$

Leche, soluciones de carboximetilcelulosa, aceite de oliva Sustitutos salivales comerciales (combinaciones de lo anterior)

Saliva autógena

Técnicas especiales de sustitución salival. Incluye un amplio abanico de posibilidades, entre las que se encuentran los dispositivos intramucosos y los depósitos en prótesis dentales, así como otros sistemas ${ }^{14,15}$ 
Tabla 4

Datos de los 29 artículos considerados para esta revisión sistemática

\begin{tabular}{|c|c|c|c|c|c|c|c|c|c|c|}
\hline Autor, año y referencia & ECC & JADAD & NE & Rec & $\mathrm{ECnC}$ & EO & SC & RS & $\mathrm{R}$ & Población de estudio \\
\hline Khurshudian, $2003^{18}$ & + & 4 & 1 & a & & & & & & P-SS \\
\hline Cassolato et al., $2003^{40}$ & & & 3 & $\mathrm{c}$ & & & & & + & P-Boca seca \\
\hline Alves et al., $2004^{19}$ & + & 3 & 1 & $\mathrm{~b}$ & & & & & & P-SS \\
\hline Gornitsky et al., $2004^{20}$ & + & 3 & 2 & $\mathrm{~b}$ & & & & & & P-pR \\
\hline Shahdad et al., $2005^{21}$ & + & 3 & 1 & $\mathrm{~b}$ & & & & & & P-pR \\
\hline McMillan et al., $2006^{22}$ & + & 3 & 2 & $\mathrm{~b}$ & & & & & & P-pR \\
\hline Taweechaisupapong et al., $2006^{23}$ & + & 3 & 1 & $\mathrm{a}$ & & & & & & P-pR \\
\hline Jham et al., $2007^{24}$ & + & 3 & 2 & $\mathrm{~b}$ & & & & & & P-pR \\
\hline McMillan et al., $2006^{35}$ & & & 2 & $\mathrm{~b}$ & & + & & & & P-pR \\
\hline Pow et al., $2006^{36}$ & & & 2 & $\mathrm{~b}$ & & + & & & & P-pR \\
\hline Ship et al., $2007^{25}$ & + & 3 & 1 & $\mathrm{a}$ & & & & & & P-SS \\
\hline Shiboski et al., $2007^{41}$ & & & 3 & $\mathrm{c}$ & & & & & + & P-pR \\
\hline Strietzel, et al., $2007^{26}$ & + & 3 & 2 & $\mathrm{~b}$ & & & & & & P-Boca seca \\
\hline Alpöz et al., $2008^{27}$ & + & 3 & 1 & $\mathrm{a}$ & & & & & & P-SS \\
\hline Yamada et al., $2007^{33}$ & & & 2 & $\mathrm{~b}$ & + & & & & & P-SS \\
\hline Turner et al., $2008^{12}$ & & & 2 & $\mathrm{a}$ & & & & + & & P-Boca seca \\
\hline Hahnel et al., $2009^{42}$ & & & 3 & c & & & & & + & P-Boca-seca \\
\hline Schiff et al., $2009^{37}$ & & & 3 & c & & + & & & & P-pR \\
\hline Kerr et al., $2010^{28}$ & + & 4 & 1 & $\mathrm{a}$ & & & & & & P-Boca seca \\
\hline Cankaya et al., $2010^{44}$ & & & 3 & c & & & + & & & P-SS \\
\hline Fedele et al., $2010^{43}$ & & & 3 & $\mathrm{c}$ & & & & & + & P-SS \\
\hline Femiano et al., $2011^{29}$ & + & 3 & 2 & $\mathrm{~b}$ & & & & & & P-Boca seca \\
\hline Antoranz et al., $2011^{13}$ & & & 3 & $c$ & & & & & + & P-Boca seca \\
\hline Iwabuchi et al., $2012^{38}$ & & & 2 & $\mathrm{~b}$ & & + & & & & P-Boca seca \\
\hline Furness et al., $2011^{39}$ & & & 1 & $\mathrm{a}$ & & & & + & & P-Boca seca \\
\hline Strietzel et al., $2011^{30}$ & + & 3 & 2 & $\mathrm{~b}$ & & & & & & P-Boca seca \\
\hline Gil Montoya, et al., $2008^{31}$ & + & 3 & 2 & $\mathrm{~b}$ & & & & & & P-Boca seca \\
\hline Silvestre et al., $2009^{34}$ & & & $2 / 3$ & c & + & & & & & P-Boca seca \\
\hline Alajbeg et al., $2012^{32}$ & + & 3 & 2 & $\mathrm{~b}$ & & & & & & P-Boca seca \\
\hline
\end{tabular}

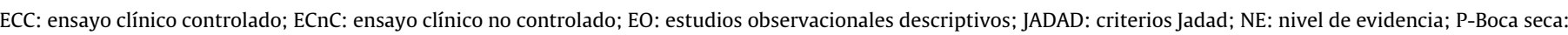

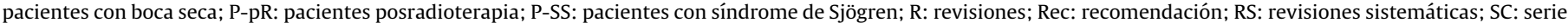
de casos.

Las palabras "dry mouth AND prognosis AND dentistry» presentaron 76 artículos que al cruzarlos con los anteriores resultaron reducidos a 4 . Así pues, después de aplicar los filtros indicados se leen a texto completo 53 artículos.

Tras la lectura se descartaron 8 por centrarse en otros temas, destacando 3 sobre el uso de la toxina botulínica, 2 acerca de su uso en hipersalivación y uno sobre el tratamiento de la hipertrofia maseterina. También se descartaron 7 revisiones que no aportaban ningún dato relevante y 9 artículos de opinión personal. Los 29 trabajos restantes, cuyos detalles más relevantes se presentan en la tabla 4 , se utilizan para la presente revisión. También se utilizan algunos artículos más antiguos y algunos que nos aportan algún aspecto de la discusión, pero no se analizan desde el punto de vista metodológico.

\section{Discusión}

Sobre la base de la revisión que hace Antoranz et al. ${ }^{13}$, agrupamos los resultados del tratamiento en etiológico-preventivo, sintomático, de estimulación salival local y tratamientos sistémicos.

\section{Tratamiento etiológico y preventivo}

Referente al tratamiento etiológico cabe destacar la importancia de determinar si la afectación glandular es definitiva o no ${ }^{12,39}$. Si nos centramos en la prevención, podemos destacar 2 trabajos que nos demuestran la utilidad de la intensity modulated radiation therapy (IMRT, «irradiación de intensidad modulada»): un estudio clínico descriptivo en 51 pacientes con cáncer nasofaríngeo que demuestra una recuperación más rápida tras el tratamiento con IMRT $^{36}$, y un estudio prospectivo longitudinal que analiza además la calidad de vida ${ }^{35}$. También merece la pena destacar el trabajo de Schiff et al. ${ }^{37}$ sobre los beneficios de la hipnosis como tratamiento preventivo de la hiposialia en los pacientes que van a ser irradiados. Es un estudio observacional prospectivo en 12 pacientes a los que se les hace una sesión de hipnosis que se graba en un $C D$ para que el paciente la escuche 2 veces al día. En los pacientes, que son evaluados hasta 12 semanas después de acabar la radioterapia, mejora la tasa salival y la sensación subjetiva de manera proporcional al número de veces que se escucha la grabación. Si bien hay trabajos que evalúan la modulación cortical que puede conseguir la hipnosis ${ }^{45}$, los propios autores citan la necesidad de hacer un ensayo aleatorizado. Finalmente, dentro de este apartado hay un interesante trabajo observacional en 323 pacientes seguidos durante un año, que relaciona la xerostomía con un incremento de enfermedades respiratorias ${ }^{38}$. Concluye, entre otros datos, que los pacientes con hiposialia tienen más enfermedades respiratorias $(\mathrm{p}<0,048)$.

\section{Tratamiento sintomático}

El objetivo prioritario es la hidratación de la cavidad oral mimetizando al máximo la saliva ${ }^{3,6}$. Se puede conseguir mediante la ingesta de líquidos ${ }^{1}$, el consumo de chicles, caramelos sin azúcar, manteniendo en la boca elementos inertes, o ingiriendo alimentos que exijan una masticación potente $e^{2,3,13}$. Si hay dolor al comer, y no se puede controlar evitando los alimentos irritantes, se puede recurrir a enjuagues con lidocaína al $2 \%^{2}$.

Hay alimentos que al contener ácidos orgánicos pueden estimular la salivación, pero perjudicar la integridad del esmalte $^{1,3,6}$. Referente a este último aspecto hay un interesante trabajo de $2011^{29}$ que alerta sobre este dato. Es un ensayo clínico controlado en paciente con boca seca, con 3 grupos de intervención (saliva artificial, ácido cítrico al 3\% y agua). Los resultados indican que si bien el sustituto salival y el ácido cítrico producen una estimulación inmediata (a los $15 \mathrm{~min}, \mathrm{p}<0,0001$ ), una hora después solo persiste la estimulación en el grupo de ácido cítrico 
(ácido cítrico frente a saliva artificial: $\mathrm{p}=0,047, \mathrm{y}$ ácido cítrico frente a placebo: $p=0,004)$. La tasa de saliva basal no mejora en ninguno de los grupos.

Si bien tenemos un abanico extenso de sustitutos salivales comercializados en forma de gel, líquido, pasta, spray, comprimidos o chicles, son pocos los trabajos que nos permiten justificar su uso. De salivas artificiales (con pocos efectos indeseables, poca duración, simulación incompleta de la saliva y ausencia de componentes bacterianos específicos) $)^{13}$ disponemos en España de Bucalsone ${ }^{\circledR}$ y Bucohidrat ${ }^{\circledR}$. Otros productos que complementan los componentes de la saliva con elementos enzimáticos son: Biotene ${ }^{\circledR}$ (gel) Oral Balance ${ }^{\circledR}$, BioXtra ${ }^{\circledR}$ y Enzycal ${ }^{\mathbb{R}}$ (todos ellos con un $\mathrm{pH}$ ácido $\mathrm{y}$, por tanto, potencialmente nocivos $)^{46}$. Con Biotene ${ }^{\circledR}$ (gel) Oral Balance ${ }^{\circledR}$ hay 2 estudios clínicos controlados, ya clásicos, realizados en pacientes con SS y en irradiados. El de Alves et al. ${ }^{19}$ es un estudio emparejado en 21 pacientes durante 90 días. Se compara con un grupo placebo y mejora la boca ardiente y la deglución, pero no mejora ni la sensación de sequedad ni la cantidad de saliva. El trabajo de Shahdad et al. ${ }^{21}$ compara Oral Balance ${ }^{\circledR}$ con BioXtra ${ }^{\circledR}$, en un estudio cruzado de 2 semanas en pacientes irradiados. Los resultados parecen favorecer al preparado BioXtra ${ }^{\circledR}$, en tanto que es más barato y más duradero el efecto. Un ensayo no controlado realizado por Silvestre et al. ${ }^{34}$ en 37 pacientes con una saliva artificial en spray (Kin Hidrat ${ }^{\mathbb{R}}$ ) obtiene que 20 de los 37 pacientes mostraron una mejoría de la sintomatología casi inmediata después de la aplicación. Por el contrario, un estudio doble ciego realizado por Gil-Montoya et al. ${ }^{31}$ en 20 ancianos institucionalizados para evaluar la efectividad de un gel oral concluye que solo mejoran algunos aspectos y que el efecto placebo debe ser considerado.

Por otro lado, McMillan et al. en $2006^{35}$, en un intento de simplificar los tratamientos, comprueban la eficacia de un dispositivo palatino con Oral Balance ${ }^{\circledR}$ frente a gel en pacientes irradiados. El dispositivo protético mantiene el producto en la boca unas $4 \mathrm{~h}$ y se muestra más efectivo para controlar los síntomas. López et al. ${ }^{14}$ publican un estudio piloto doble ciego en el que no obtienen diferencias significativas entre Biotene ${ }^{\circledR}$ y agua ${ }^{14}$.

Una modificación perfeccionando los reservorios, caros e incómodos, se muestra en un estudio clínico controlado, doble ciego, en 27 pacientes, para evaluar el uso de discos OraMoist ${ }^{\circledR}$ frente a discos placebo colocados de forma adhesiva en el paladar $^{28}$. Los discos se disuelven en unas $2-4 \mathrm{~h}$ y no interfieren ni en el habla ni en la comida. Como resultados podemos destacar que su uso mejora la cantidad de saliva y el estado subjetivo del paciente (iniciándose a los 60 min y perdurando más de $4 \mathrm{~h}$ ).

Otra línea de productos existente para aliviar la sequedad bucal es la línea Xerostom ${ }^{\circledR}$ (no contiene detergentes, por lo que no irrita la mucosa; el pH es neutro y tiene múltiples presentaciones). Está compuesto por aceite de oliva, betaína, flúor y xilitol. El flúor y el xilitol controlan la producción de caries y el aceite de oliva es útil en el control de la proliferación bacteriana ${ }^{47}$. Es, además, el único producto de este tipo en el que hemos encontrado un ensayo clínico controlado realizado en 40 pacientes con boca seca polimedicados ${ }^{25}$. Otros estudios, menos relevantes, han obtenido buenos resultados con $\operatorname{Xeros}^{\circledR}$ (contiene betaína, además de aloe vera y ácido málico, fluoruro sódico y xilitol $)^{48}$, o con Kin Hidrat ${ }^{\circledR}$ (contiene ácido cítrico, flúor y xilitol). Otro de los productos sustitutivos bien evaluado, no comercializado en España, es el XialineTM ${ }^{\circledR}$ (un sustituto salival basado en el biopolímero natural goma de xantano). Alpöz et al. ${ }^{27}$ desarrollan un estudio doble ciego en 29 pacientes con SS. Se mide el flujo salival y las quejas subjetivas de los pacientes. A un grupo se le aplica el producto, y al otro, placebo ( preparado con agua del grifo y té diluido con el fin de asemejarlo a Xialine ${ }^{\circledR}$ ). Es un estudio emparejado de 14 días, una semana de descanso y a los 14 días se les adscribe al otro grupo. En general, los pacientes prefirieron Xialine ${ }^{\circledR}$ al final del estudio $(p=0,011)$, pero los efectos por separado no muestran diferencias estadísticamente significativas. El efecto en el grupo tratamiento se inicia antes y se mantiene más tiempo.

\section{Estimulación local}

Si el parénquima salival es funcionante podemos estimularlo de manera mecánica, quimicagustativa y eléctrica. Los 2 primeros métodos ya los hemos comentado $2,3,6,13,29$. En lo referente a la electroestimulación ${ }^{26,39}$, la Food and Drug Administration (FDA) aprobó en 1988 el uso del Biosonics ${ }^{\circledR}$. Se trata de una boquilla conectada con un cable a un módulo de control. Se colocaba unos minutos en el dorso lingual mejorando el flujo salival, pero resultaba aparatosa y cara $^{49}$. Posteriormente se fabricó el neuroestimulador Saliwel GenNarino ${ }^{\mathbb{R}}$; simula una férula de descarga adaptada a la mandíbula con un dispositivo electrónico en miniatura situado en la mucosa del cordal, permite la estimulación del nervio lingual e incluso del bucal a una distancia de 1 a $5 \mathrm{~mm}$ y se regula por control remoto ${ }^{26,30,50}$. Más recientemente, el Saliwel Crown ${ }^{\circledR}$ es un dispositivo en miniatura adaptado a la corona de un implante colocado en el área del tercer molar ${ }^{43}$. Si bien muchos autores citan la bondad de estos sistemas $^{13,26,43}$, la revisión Cochrane Collaboration ${ }^{\circledR}$ de $2011^{39}$ menciona un único artículo de $2007^{26}$ y lo excluye de su revisión, a pesar de ser un estudio piloto en 23 pacientes. Posteriormente 2 trabajos publicados, en base al proyecto Saliwell y el aparato GenNarino ${ }^{\circledR 30,32}$, lo presentan como un método prometedor para los pacientes polimedicados. En el primero, multicéntrico con 14 centros, 13 países y 114 pacientes, se hace un primer estudio piloto doble ciego de un mes, y luego un estudio abierto de 9 meses con una primera evaluación a los 5 meses. Obtiene una $\mathrm{p}<0,05$ tanto para los valores subjetivos como para los objetivos.

\section{Estimulación sistémica}

La pilocarpina, medicamento parasimpático, continúa siendo uno de los productos más estudiados. Su principal problema es que hasta un 30\% de los pacientes sufren efectos adversos. Su dosis es de 5-10 mg 3-4 veces al día ${ }^{13}$. Entre sus contraindicaciones claras está el glaucoma, el asma incontrolada y la úlcera gástrica ${ }^{2}$. Hay 2 trabajos que evalúan su actuación en pacientes irradiados por cáncer de cabeza y cuello. El de Gornitsky et al. ${ }^{20}$ evalúa su utilidad en un estudio de 58 pacientes que recibieron radioterapia en ambas parótidas. En una primera fase ( 4 semanas de duración de la radioterapia) se tomó 5 veces al día. En una segunda fase del estudio, 5 semanas posradioterapia, se tomó 4 veces al día. Se observó una mejora en la calidad de vida y una disminución en el nivel de incomodidad en la primera fase del estudio. No se mostró efectiva para incrementar la producción de saliva y los resultados de la segunda fase son difíciles de precisar, entre otras razones por el alto grado de incumplimiento. El segundo trabajo es un ensayo clínico en paralelo, también en pacientes irradiados, que compara comprimidos de Salagen ${ }^{\circledR}$ (5 mg de pilocarpina en comprimidos que se ingieren con un vaso de agua) y de 3 y $5 \mathrm{mg}$ de pilocarpina (comprimidos para disolver en boca) con placebo. Los mejores resultados se obtienen con los comprimidos de $5 \mathrm{mg}$ de pilocarpina y no se presentan efectos adversos destacables ni modificaciones significativas de la presión arterial entre los diferentes grupos ${ }^{23}$.

Otro estimulante salival, también aceptado por la FDA, es la cevimelina (agonista colinérgico). Yamada et al. ${ }^{33}$, en un ensayo clínico no controlado en 33 mujeres diagnosticadas de SS, indican que cuanto mayor es el grado de sialectasias y de infiltrado linfocitario periductal, peor es la respuesta salival conseguida. Por eso recomiendan que antes de utilizar esta medicación, no exenta de complicaciones, hagamos un diagnóstico de la gravedad de la enfermedad mediante sialografía y biopsia de labio. 
Otro trabajo que también evalúa una acción sistémica más novedosa, esta vez en pacientes diagnosticadas de SS primario, es el de Cankaya et al. ${ }^{44}$. Los autores presentan una serie de 30 mujeres tratadas con hidroxicloroquina $(400 \mathrm{mg} / \mathrm{d}$, repartidos en 2 dosis). Se evalúa la tasa de saliva en reposo y estimulada, así como parámetros subjetivos. Los controles se realizan cada 6 semanas (hasta la 30) y los resultados no resultan significativos.

Jham et al. ${ }^{24}$ analizan la producción salival en un ensayo clínico controlado en 55 pacientes con cáncer de cabeza y cuello que iban a ser irradiados. Utilizan betanecol $25 \mathrm{mg} 3$ veces al día desde el inicio de la radioterapia en comparación con un grupo control. Al grupo control se le administró Oral Balance ${ }^{\circledR}$ con la idea de mejorar su sintomatología y asumiendo que no tiene efectos sobre la producción salival. Consiguen una mejora importante de la salivación de manera inmediata, pero no resulta significativa a medio plazo, después de acabar la radioterapia. La sensación subjetiva no mejora significativamente, si bien los autores no tienen en cuenta el posible sesgo que el uso de la saliva artificial puede representar. Creemos, por otro lado, que sería necesario comparar sus resultados y efectos adversos en un estudio controlado con pilocarpina.

Finalmente, dentro de este apartado comentamos un estudio doble ciego ${ }^{18}$. Se utiliza interferón- $\alpha, 150$ UI, 3 veces al día, en 8 pacientes frente a 4 del grupo control $(200 \mathrm{mg}$ de excipiente conteniendo maltosa y estearato de magnesio al $0,5 \%$ ), todos ellos con SS. Se hizo un seguimiento de 24 semanas y 5 de los pacientes del grupo estudio continuaron en un ensayo abierto 24 semanas más. Los resultados resultaron estadísticamente significativos para el incremento de saliva y lagrimeo, así como para la mejora subjetiva. Estos resultados no concuerdan con el trabajo más antiguo descrito por Ship et al. ${ }^{51}$, en el que informaron de que en 12 semanas de seguimiento las tasas de saliva estimulada aumentaron en el grupo que recibió 150 UI de interferón 3 veces al día, pero no alcanzaron significación estadística.

\section{Conclusiones}

El pronóstico de la sequedad bucal está condicionado por su prevención, así como por el diagnóstico preciso de las causas y el tratamiento precoz. El objetivo primordial es conseguir una buena hidratación. El tratamiento no siempre obtiene resultados satisfactorios.

Debido a su etiología multifactorial, el tratamiento debe ser individualizado, empleando desde las técnicas más sencillas para estimular la producción (masticar chicles o caramelos), hasta las complejas (pilocarpina o cevilemina, por ejemplo) o las más sofisticadas, como el Saliwell Crown ${ }^{\circledR}$. La pilocarpina continúa siendo un buen tratamiento sistémico.

Hay 2 productos de uso tópico con buenos estudios que parecen avalar su uso: Oral Balance ${ }^{\mathbb{R}}$ y Xerostom ${ }^{\mathbb{R}}$.

La electroestimulación está reportando buenos resultados y parece estar especialmente indicada en los pacientes polimedicados. Es un método complejo y caro.

\section{Conflicto de intereses}

Los autores declaran no tener ningún conflicto de intereses.

\section{Bibliografía}

1. Chimenos-Küstner E, Ferrer-Benaiges M, Poirier-Aldea C, López-López J, Caballero-Herrera R. Xerostomía: diagnóstico y tratamiento. Anales Odontoestomatol. 1997; $1: 30-3$

2. Silvestre-Donat FJ, Miralles-Jordá L, Martínez-Mihi V. Tratamiento de la boca seca: puesta al día. Med Oral. 2004;9:273-9.

3. Chimenos E, Marques MS. Boca ardiente y saliva. Med Oral. 2002;7:244-53.

4. Jiménez Duarte J. Aspectos clínicos y tratamiento de la xerostomía. Acta Otorrinolaringol \& Cir Cab-Cuello. 2005;33:14-20.
5. Sreebny LM. Saliva in health and disease: An appraisal and update. Int Dent J. 2000;50:140-61.

6. Bascones A, Tenovuo J, Ship J, Turner M, Mac-Veigh I, López-Ibor JM, et al. Conclusiones del Simposium 2007 de la Sociedad Española de Medicina Oral sobre "Xerostomía. Síndrome de Boca Seca. Boca Ardiente». Av Odontoestomatol. 2007;23:119-26.

7. Ship J, Fox PC, Baum BJ. How much saliva is enough? 'Normal' function defined. J Am Dent Assoc. 1991;122:63-9.

8. Agar M, Currow D, Plummer J, Seidel R, Carnahan R, Abernethy AP. Changes in anticholinergic load from regular prescribed medications in palliative care as death approaches. Palliat Med. 2009;23:257-65.

9. Soares MS, Chimenos-Küstner E, Subirá-Pifarrè C, Rodríguez de Rivera-Campillo ME, López-López J. Association of burning mouth syndrome with xerostomia and medicines. Med Oral Patol Oral Cir Bucal. 2005;10:301-8.

10. James L. Milk protein and the restoration of fluid balance after exercise. Med Sport Sci. 2012:59:120-6.

11. McGrail A, Kelchner LN. Adequate oral fluid intake in hospitalized stroke patients: Does viscosity matter? Rehabil Nurs. 2012;37:252-7.

12. Turner M, Jahangiri L, Ship JA. Hyposalivation, xerostomia and the complete denture: A systematic review. J Am Dent Assoc. 2008;139:146-50.

13. Antoranz A, Casado L, Corral C, Cerero R, López P. Pacientes con xerostomía: un reto terapéutico para el odontoestomatólogo. Cient Dent. 2011;8:213-7.

14. López López J, Roselló Llabrés X, Blanco Carrión A. El uso de reservorios de saliva en la terapéutica de la xerostomia. Revista Europea de Odontoestomatología. 1995; 7:283-8.

15. Agrawal KK, Singh SV, Rashmikant US, Singh RD, Chand P. Prosthodontic rehabilitation in Sjogren's syndrome with a simplified palatal reservoir: Two year follow up. J Indian Prosthodont Soc. 2010;10:249-52.

16. Ebell MH, Siwek J, Weiss BD, Woolf SH, Susman J, Ewigman B, et al. Strength of recommendation taxonomy (SORT): A patient-centered approach to grading evidence in the medical literature. J Am Board Fam Pract. 2004;17:59-67.

17. Jadad AR, Moore RA, Carroll D, Jenkinson C, Reynolds DJ, Gavaghan DJ, et al. Assessing the quality of reports of randomized clinical trials: Is blinding necessary? Control Clin Trials. 1996;17:1-12.

18. Khurshudian AV. A pilot study to test the efficacy of oral administration of interferon-alpha lozenges to patients with Sjögren's syndrome. Oral Surg Oral Med Oral Pathol Oral Radiol Endod. 2003;95:38-44.

19. Alves MB, Motta AC, Messina WC, Migliari DA. Saliva substitute in xerostomic patients with primary Sjögren's syndrome: A single-blind trial. Quintessence Int. 2004;35:392-6.

20. Gornitsky M, Shenouda G, Sultanem K, Katz H, Hier M, Black M, et al. Doubleblind randomized, placebo-controlled study of pilocarpine to salvage salivary gland function during radiotherapy of patients with head and neck cancer. Oral Surg Oral Med Oral Pathol Oral Radiol Endod. 2004:98:45-52.

21. Shahdad SA, Taylor C, Barclay SC, Steen IN, Preshaw PM. A double-blind, crossover study of Biotène Oralbalance and BioXtra systems as salivary substitutes in patients with post-radiotherapy xerostomia. Eur J Cancer Care (Engl). 2005:14:319-26.

22. McMillan AS, Pow EH, Kwong DL, Wong MC, Sham JS, Leung LH, et al. Preservation of quality of life after intensity-modulated radiotherapy for earlystage nasopharyngeal carcinoma: Results of a prospective longitudinal study. Head Neck. 2006;28:712-22.

23. Taweechaisupapong S, Pesee M, Aromdee C, Laopaiboon M, Khunkitti W. Efficacy of pilocarpine lozenge for post-radiation xerostomia in patients with head and neck cancer. Aust Dent J. 2006;51:333-7.

24. Jham BC, Teixeira IV, Aboud CG, Carvalho AL, Coelho Mde M, Freire AR. A randomized phase III prospective trial of bethanechol to prevent radiotherapyinduced salivary gland damage in patients with head and neck cancer. Oral Oncol. 2007;43:137-42

25. Ship JA, McCutcheon JA, Spivakovsky S, Kerr AR. Safety and effectiveness of topical dry mouth products containing olive oil, betaine, and xylitol in reducing xerostomia for polypharmacy-induced dry mouth. J Oral Rehabil 2007;34:724-32.

26. Strietzel FP, Martin-Granizo R, Fedele S, Lo Russo L, Mignogna M, Reichart PA, et al. Electrostimulating device in the management of xerostomia. Oral Dis. 2007; 13:206-13.

27. Alpöz E, Güneri P, Onder G, Cankaya H, Kabasakal Y, Köse T. The efficacy of Xialine in patients with Sjögren's syndrome: A single-blind, cross-over study. Clin Oral Investig. 2008:12:165-72.

28. Kerr AR, Corby PM, Shah SS, Epler M, Fisch GS, Norman RG. Use of a mucoadhesive disk for relief of dry mouth: A randomized, double-masked, controlled crossover study. J Am Dent Assoc. 2010;141:1250-6.

29. Femiano F, Rullo R, di Spirito F, Lanza A, Festa VM, Cirillo N. A comparison of salivary substitutes versus a natural sialogogue (citric acid) in patients complaining of dry mouth as an adverse drug reaction: A clinical, randomized controlled study. Oral Surg Oral Med Oral Pathol Oral Radiol Endod. 2011;112:e15-20.

30. Strietzel FP, Lafaurie GI, Mendoza GR, Alajbeg I, Pejda S, Vuletić L, et al. Efficacy and safety of an intraoral electrostimulation device for xerostomia relief: A multicenter, randomized trial. Arthritis Rheum. 2011;63:180-90.

31. Gil-Montoya JA, Guardia-López I, González-Moles MA. Evaluation of the clinical efficacy of a mouthwash and oral gel containing the antimicrobial proteins lactoperoxidase, lysozyme and lactoferrin in elderly patients with dry mouth-A pilot study. Gerodontology. 2008;25:3-9.

32. Alajbeg I, Falcão DP, Tran SD, Martín-Granizo R, Lafaurie GI, Matranga D, et al. Intraoral electrostimulator for xerostomia relief: A long-term, multicenter, 
open-label, uncontrolled, clinical trial. Oral Surg Oral Med Oral Pathol Oral Radiol Endod. 2012;113:773-81.

33. Yamada H, Nakagawa Y, Wakamatsu E, Sumida T, Yamachika S, Nomura Y, et al. Efficacy prediction of cevimeline in patients with Sjögren's syndrome. Clin Rheumatol. 2007;26:1320-7.

34. Silvestre FJ, Minguez MP, Suñe-Negre JM. Clinical evaluation of a new artificial saliva in spray form for patients with dry mouth. Med Oral Patol Oral Cir Bucal. 2009;1:e8-11.

35. McMillan AS, Tsang CS, Wong MC, Kam AY. Efficacy of a novel lubricating system in the management of radiotherapy-related xerostomia. Oral Oncol. 2006;42:842-8.

36. Pow EH, Kwong DL, McMillan AS, Wong MC, Sham JS, Leung LH, et al. Xerostomia and quality of life after intensity-modulated radiotherapy vs. conventional radiotherapy for early-stage nasopharyngeal carcinoma: Initial report on a randomized controlled clinical trial. Int J Radiat Oncol Biol Phys. 2006;66: 981-91.

37. Schiff E, Mogilner JG, Sella E, Doweck I, Hershko O, Ben-Arye E, et al. Hypnosis for postradiation xerostomia in head and neck cancer patients: A pilot study. J Pain Symptom Manage. 2009;37:1086-92.

38. Iwabuchi H, Fujibayashi T, Yamane GY, Imai H, Nakao H. Relationship between hyposalivation and acute respiratory infection in dental outpatients. Gerontology. 2012;58:205-11.

39. Furness S, Worthington HV, Bryan G, Birchenough S, McMillan R. Interventions for the management of dry mouth: Topical therapies. Cochrane Database Syst Rev. 2011;12:CD008934.

40. Cassolato SF, Turnbull RS. Xerostomia: Clinical aspects and treatment. Gerodontology. 2003;20:64-77.

41. Shiboski CH, Hodgson TA, Ship JA, Schiødt M. Management of salivary hypofunction during and after radiotherapy. Oral Surg Oral Med Oral Pathol Oral Radiol Endod. 2007;103 Suppl:S66.e1-1.
42. Hahnel S, Behr M, Handel G, Bürgers R. Saliva substitutes for the treatment of radiation-induced xerostomia-A review. Support Care Cancer. 2009;17: 1331-43.

43. Fedele S, Wolff A, Strietzel FP, Martín-Granizo López R, Porter S, Konttinen YT. Electrostimulation for the treatment of dry mouth. Harefuah. 2010;149: 99-103.

44. Cankaya H, Alpöz E, Karabulut G, Güneri P, Boyacioglu H, Kabasakal Y. Effects of hydroxychloroquine on salivary flow rates and oral complaints of Sjögren patients: A prospective sample study. Oral Surg Oral Med Oral Pathol Oral Radiol Endod. 2010;110:62-7.

45. Faymonville ME, Laureys S, Degueldre C, DelFiore G, Luxen A, Franck G, et al. Neural mechanisms of antinociceptive effects of hypnosis. Anesthesiology. 2000;92:e1257-6

46. Hannig C, Spitzmüller B, Lux HC, Altenburger M, Al-Ahmad A, Hannig M Efficacy of enzymatic toothpastes for immobilisation of protective enzymes in the in situ pellicle. Arch Oral Biol. 2010;55:463-9.

47. Wiegand A, Gutsche M, Attin T. Effect of olive oil and an olive-oil-containing fluoridated mouthrinse on enamel and dentin erosion in vitro. Acta Odontol Scand. 2007;65:357-61.

48. Söderling E, Le Bell A, Varpuleena K, Tenovuo J. Betaine-containing toothpaste relieves subjective symptoms of dry mouth. Acta Odontol Scand. 1998;56:65-9.

49. Ami S, Wolff A. Implant-supported electrostimulating device to treat xerostomia: A preliminary study. Clin Implant Dent Relat Res. 2010;12:62-71.

50. Kruize AA, Hené RJ, Kallenberg CG, van Bijsterveld OP, van der Heide A, Kater L, et al. Hydroxychloroquine treatment for primary Sjögren's syndrome: A two year double blind crossover trial. Ann Rheum Dis. 1993;52:360-4.

51. Ship JA, Fox PC, Michalek JE, Cummins MJ, Richards AB, The IFN protocol study group. Treatment of primary Sjogren's syndrome with low-dose natural human interferon-alpha administered by the oral mucosal route: A phase II clinical trial. J Interferon Cytokine Res. 1999;19:943-51. 